\title{
CERTIFICAÇÃO AMBIENTAL DE HABITAÇ̃̃ES LEED E AS MUDANÇAS NA GESTÃO DA CONSTRUÇÃO CIVIL SUSTENTÁVEL NA AMÉRICA LATINA
}

\section{ENVIRONMENTAL HOUSING CERTIFICATION LEED AND IMPROVED PROJECT MANAGEMENT OF SUSTAINABLE CONSTRUCTION IN LATIN AMERICA}

\section{Cristina Shoji Pellizzetti, Especialista (Middlesex University London).}

\section{Palavras Chave}

Certificação ambiental; LEED for homes; Construção sustentável; América Latina; Sustentabilidade.

\section{Key Words}

Environmental certification; LEED for homes; Green Building, Latin America, Sustainability.

\section{RESUMO}

Edificações de alto desempenho certificadas pelo sistema de certificação ambiental internacional LEADERSHIP IN ENERGY AND ENVIRONMENTAL DESIGN (LEED), outorgado pela United States Green Buiding Council (USGBC) dos Estados Unidos, garantem ao cliente que o seu investimento foi empregado conforme planejado significando menores riscos, melhor eficiência energética e hídrica dos sistemas instalados, redução dos custos operacionais, qualidade dos ambientes internos e bem estar do usuário, durante o ciclo de vida da habitação. Desde 1998, mais de 34.700 empreendimentos comerciais e 110.370 unidades residenciais já receberam a certificação LEED em 162 países. Em 2015 na América Latina, duas residências foram certificadas na versão residencial LEED FOR HOMES no Brasil e Colômbia. No Chile, Peru, Costa Rica e México, outros 23 projetos residenciais registrados estão em construção. Sistemas de certificação ambiental de empreendimentos visam incentivar a transformação e sustentabilidade na indústria desde a concepção do projeto e durante o ciclo de vida de uma edificação.

\section{ABSTRACT}

High performance buildings certified by the international environmental certification system LEADERSHIP IN ENERGY AND DESIGN ENVIRONMENTAL (LEED), granted by the United States Green Building Council (USGBC) guarantee to the homeowner that their investment was employed as planned meaning less risks, improved energy and water efficiency of systems installed, reducing operating costs, increasing quality of indoor and welfare of the homewoner, during the housing lifecycle. Since 1998, over 34,700 LEED commercial e 110,370 residencial units, have received LEED certification in 162 countries. In 2015 in Latin America, two single family homes have been certified in the residential version LEED FOR HOMES in Brazil and Colombia. In Chile, Peru, Costa Rica and Mexico another 23 residential projects are under construction. Environmental certification systems developments aim to encourage the transformation and sustainability in the industry from the design of the project and during the life cycle of a building. 


\section{INTRODUÇÃO}

Sistemas de certificação ambiental de empreendimentos visam incentivar a transformação e sustentabilidade na indústria desde a concepção do projeto, até a sua implantação, buscando soluções e métodos construtivos que reduzam o impacto causado pela construção civil ao meio ambiente, além de eficiência energética, qualidade dos ambientes internos e bem estar do usuário, durante o ciclo de vida de uma edificação.

Desde o lançamento do sistema de certificação ambiental norte-americano Leadership in Energy and Environmental Design - LEED em 1998 pela organização norte-americana United States Green Building Council USGBC, mais de 82.800 projetos comerciais e 245.541 unidades habitacionais seguem as diretrizes de gestão de projeto e obra exigidos para o atendimento LEED no mundo. São torres de escritórios, shoppings centers, galpões logísticos, data centers, instalações esportivas, museus, instituições de ensino, unidades de saúde, bibliotecas, agências bancárias, supermercados, lojas de varejo, estádios de futebol, residências e até bairros sustentáveis.

De acordo com a organização local no Brasil, em 2016 o país mantém a $5^{\text {a }}$ posição no ranking mundial de empreendimentos LEED, com 380 empreendimentos certificados e um total de 1.156 registros, atrás dos Estados Unidos, China, Emirados Árabes Unidos e Índia.

Como exemplo do que se observou nos Estados Unidos e na Europa, primeiro as certificações ambientais foram concedidas às edificações comerciais e, posteriormente, houve o interesse da aplicação a edifícios habitacionais. Nessa linha, a certificação ambiental de habitações LEED FOR HOMES criada em 2008 especificamente para edificações residenciais já certificou mais de 110.370 unidades habitacionais nos EUA, Canada, Arábia Saudita, Montenegro, Hong Kong, China, Turquia.

Na América Latina, em 2015 duas residências receberam a certificação ambiental de habitações LEED FOR HOMES, sendo uma no Brasil e outra na Colômbia. A aplicação dos processos estruturados do sistema norte-americano LEED na América Latina exige novas atividades de gestão de design, liderados pelo arquiteto e empresa construtora, assim como o empenho colaborativo de toda a equipe, desenvolvimento de simulação energética computacional na fase projeto, vistorias presenciais durante a construção e a necessidade de disponibilizar informações técnicas dos produtos por parte dos fornecedores.

A obtenção da certificação ambiental de habitações garante ao proprietário que o seu investimento foi empregado conforme planejado significando menores riscos, melhor eficiência energética e hídrica dos sistemas instalados, redução dos custos operacionais, qualidade dos ambientes internos e bem estar do usuário, durante o ciclo de vida da habitação.

\section{REFERENCIAIS E PROPOSIÇÕES TEÓRICAS}

Sustentabilidade na construção civil pode ser resumida como medidas construtivas e procedimentos que buscam aumentar a eficiência de um empreendimento no uso dos recursos naturais, com foco na redução dos impactos ambientais, econômicos e sociais que este pode ocasionar durante todo o seu ciclo de vida (Arbache, 2010).

A expressão Greenbuilding foi cunhada para englobar todas as iniciativas dedicadas à criação de construções que utilizem recursos de maneira eficiente, com claro foco em uso de energia; que sejam confortáveis; e que tenham maior longevidade, adaptando-se às mudanças nas necessidades dos usuários e permitindo desmontagem ao final do ciclo de vida do edifício, para aumentar a vida útil dos componentes através de sua reutilização ou reciclagem (Vanessa Gomes, 2003).

O presente artigo demonstra ser o intuito da certificação voluntária LEED FOR HOMES outorgado pela organização americana United States Green Building Council - USGBC, o de oferecer diretrizes às equipes na fase de concepção e integrar os programas durante a construção, agregando valor de mercado estabelecido através da legitimidade de um processo específico validado por uma terceira parte independente.

\section{DESENVOLVIMENTO DO CASE}

\subsection{Sistema de certificação ambiental interna- cional LEED}

O United States Green Building Council - USGBC , é uma organização não governamental, criada em 1993 em Washington D.C., nos Estados Unidos. O objetivo foi de incentivar a transformação e sustentabilidade na indústria desde a concepção do projeto, até a sua implantação, buscando soluções e métodos construtivos que reduzam o impacto causado pela construção civil ao meio ambiente, além de eficiência energética, qualidade dos ambientes internos e bem estar do usuário, durante o ciclo de vida de uma edificação. A organização é responsável pela análise documental das práticas de sustentabilidade ambiental e pela outorga da certificação ambiental internacional LEED a empreendimentos em 162 países. 
Figura 1: U.S. Green Building Council \& LEED \& LEED FOR HOMES. Fonte: "USGBC"
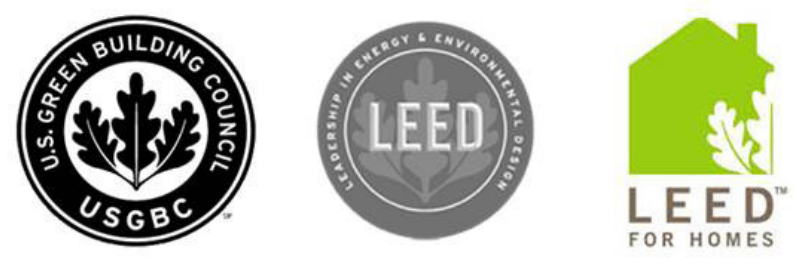

Fonte: "USGBC"

O Leadership in Energy and Environmental Design LEED é uma ferramenta de avaliação desenvolvida pela USGBC em 1998, sendo atualmente o mais reconhecido no mercado brasileiro e no mundo.

Para que a edificação receba a certificação ambiental de habitações LEED FOR HOMES (LEED BD+C: Homes v3 - LEED 2008) é necessário cumprir pré-requisitos e créditos comprovando a adoção de práticas de sustentabilidade ambiental, creditados ao projeto em oito categorias: Inovação e Projeto (ID), Localização e Transporte ( LL), Implantação Sustentável ( SS ), Uso Racional da Água ( WE), Energia e Atmosfera ( EA), Materiais e Recursos ( MR), Qualidade Ambiental Interna (EQ) e Práticas Sociais ( $A E$ ).

O nível da certificação é definido conforme a quantidade de pontos adquiridos na avaliação nas oito categorias e 136 créditos, podendo variar de nível Certificado, Prata, Ouro ou Platina, verificando a possibilidade de atendimento aos 18 pré-requisitos obrigatórios e ao número mínimo de 40 pontos.

Figura 2: Níveis de certificação. Fonte: "USGBC"

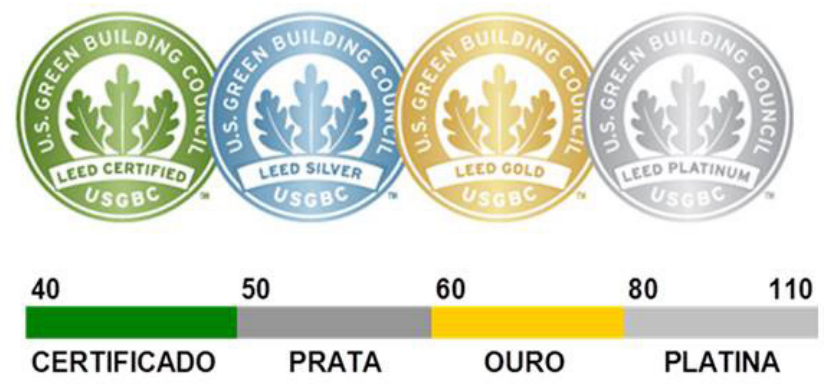

Fonte: "USGBC"

O LEED é um guia de certificação ambiental para empreendimentos sustentáveis e o PMBOK é um guia de gerenciamento de projetos, ambos reconhecidos internacionalmente. O LEED aborda a obrigatoriedade do gerenciamento do projeto e obra a partir de processos estruturados de construção sustentável compatíveis com áreas de conhecimento do PMBOK (Gerenciamento da Integração, Escopo, Tempo, Custos, Qualidade, Recursos
Humanos, Riscos, Aquisições, Comunicações) seguindo o grupo de processos ( Iniciação, Planejamento, Execução, Monitoramento e Controle, Encerramento) .

Desde o lançamento da versão residencial LEED FOR HOMES em 2008 especificamente para edificações residenciais com 392 projetos pilotos nos Estados Unidos, o número evoluiu para mais de 245.541 unidades habitacionais registradas na sua maioria nos Estados Unidos, Canadá e Emirados Árabes.

\subsection{Processo LEED FOR HOMES}

A aplicação dos processos estruturados do sistema de certificação ambiental internacional de habitações LEED FOR HOMES na construção sustentável na América Latina e especificamente no Brasil exige a liderança de um gestor integrador para o desenvolvimento de um processo integrado desde o início do processo de design e durante a obra.

A certificação é outorgada pela organização United States Green Building Council - USGBC sediada nos Estados Unidos, através da análise documental das práticas de sustentabilidade ambiental adotadas pelo empreendimento na fase projeto e construção, apresentadas na conclusão do empreendimento.

O processo de certificação ambiental de habitações LEED FOR HOMES tem inicio com o registro do projeto on-line a partir da plataforma https://www.usgbc.org/ leedonline/ . Durante a fase projeto, diretrizes para atendimento ao processo de certificação são determinadas em um processo integrado colaborativo de todos os envolvidos. $\mathrm{O}$ auditor local ( Green Rater) é responsável pelas verificações in loco na obra, obrigatoriamente. Após conclusão da obra, a documentação comprobatória de atendimento aos pré-requisitos e créditos será revisada pela empresa provedora ( Provider) dos Estados Unidos e testes de performance serão realizados após a obra concluída e os resultados serão submetidos à organização americana USGBC. O proprietário responsável pelo projeto receberá o anúncio da conclusão do processo e obtenção da certificação ambiental com a pontuação obtida, juntamente com o certificado, que indicam o mérito alcançado.

\subsubsection{Equipe técnica LEED FOR HOMES}

A equipe técnica do processo de Auditoria LEED FOR HOMES é formada por empresa provedora (Provider) dos Estados Unidos e profissional com a função de auditor local (Green Rater), credenciados pela organização USGBC. Os chamados profissionais LEED Accredited Professionals - LEED APs são consultores especialistas que auxiliam a 
equipe de projeto e obra no processo e na obtenção da certificação. No mundo são mais de 200 mil profissionais credenciados, na sua maioria arquitetos e engenheiros.

Contratante - Responsável pela condução geral da certificação e engajamento de todos os envolvidos, em todas suas fases;

Arquiteto - Profissional responsável pelo desenvolvimento dos Projetos de Arquitetura e Paisagístico;

Engenheiros e Especialistas - Profissionais responsáveis pelo desenvolvimento dos Projetos Técnicos;

Construtora - Empresa responsável pela execução da construção, gerenciamento da gestão de resíduos e capacitação dos trabalhadores;

Consultor LEED- Profissional responsável pelo auxílio à equipe de projeto e obra no processo de certificação ambiental internacional de habitações LEED FOR HOMES;

USGBC - United States Green Building Council Organização americana responsável pela análise documental das práticas de sustentabilidade ambiental adotadas pelo empreendimento e pela outorga da certificação ambiental internacional de habitações LEED for Homes

Empresa provedora ( PROVIDER) - Empresa americana responsável pela supervisão do processo de Auditoria LEED FOR HOMES e comunicação com a USGBC.

Auditor local ( GREEN RATER) - Profissional local responsável pela inspeção do processo de Auditoria LEED FOR HOMES e comunicação com a empresa americana Provider.

\subsubsection{Critérios para certificação LEED FOR HOMES}

Para que um imóvel receba a certificação ambiental internacional de habitações LEED FOR HOMES na conclusão da obra é necessário cumprir 18 pré-requisito obrigatórios e até 136 créditos nas oito categorias, comprovando a adoção de práticas de sustentabilidade ambiental, creditados ao projeto de acordo com as recomendações estabelecidas pelo sistema LEED BD+C: Homes v3 - LEED 2008.

\subsubsection{Categorias, pré-requisitos e créditos LEED FOR HOMES}

Pré-requisitos são requisitos mínimos obrigatórios, a serem atendidos pelo projeto para que o mesmo tenha direito a acumulação de pontos para certificação, caso não sejam atendidos o projeto não poderá ser certificado.
Figura 3: Pontuação LEED FOR HOMES.

\begin{tabular}{|c|c|c|c}
\hline $\begin{array}{c}\text { Categorias LEED FOR HOMES } \\
\text { ( LEED BD+C: Homes v3 - LEED 2008) } \\
\text { *A nova versão LEED FOR HOMES v4 será } \\
\text { obrigatória a partir de Outubro 2016 }\end{array}$ & $\begin{array}{c}\text { Pré } \\
\text { requisitos }\end{array}$ & $\begin{array}{c}\text { Mínimo } \\
\text { pontos }\end{array}$ & $\begin{array}{c}\text { Máximo } \\
\text { pontos }\end{array}$ \\
\hline $\begin{array}{l}\text { Inovação e Projeto ( ID ) } \\
\text { Localização e Transporte ( LL) }\end{array}$ & 3 & 0 & $\mathbf{1 1}$ \\
\hline Implantação Sustentável ( SS) & 0 & 0 & $\mathbf{1 0}$ \\
\hline Uso Racional da Água (WE) & 2 & 5 & $\mathbf{2 2}$ \\
\hline Energia e Atmosfera ( EA) & 2 & 3 & $\mathbf{1 5}$ \\
\hline Materiais e Recursos (MR) & 3 & 2 & $\mathbf{3 8}$ \\
\hline Qualidade Ambiental Interna ( EQ) & 7 & 6 & $\mathbf{2 1}$ \\
\hline Práticas Sociais ( AW) & 1 & 0 & $\mathbf{3}$ \\
\hline \multicolumn{1}{c}{ TOTAL } & $\mathbf{1 8}$ & $\mathbf{1 6}$ & $\mathbf{1 3 6}$ \\
\hline
\end{tabular}

Fonte: "elaborada pelos autores"

\subsection{Créditos de Inovação Tecnológica e Ambiental A. Categorias de INOVAÇÃO E PROJETO (ID)}

A categoria premia o Processo de Projeto Integrado e estimula o trabalho multidisciplinar dos vários profissionais envolvidos desde o início do processo de design e durante a obra.

Pré-requisitos (ID):

ID 1.1. Elaborar um estudo de viabilidade da certificação LEED FOR HOMES

ID 2.1. Implantar um plano de Gerenciamento da Qualidade

ID 2.2. Implantar um plano de Gerenciamento da Durabilidade

Créditos (ID) :

ID 1.2. Projeto Integrado, Planejamento

ID 1.3. Profissional credenciado LEED APs LEED FOR HOMES

ID 1.4. Projeto integrado, Atividades

ID 1.5. Orientação de Projeto - Carta Solar

ID 2.3. Comissionamento do gerenciamento da qualidade ID 3.1 A 3.4. Inovação e Projeto

\section{B. Categoria LOCALIZAÇÃO E TRANSPORTE (LL)}

A categoria incentiva e recompensa opções de construção em locais previamente desenvolvidos e promove o desenvolvimento de bairros tranquilos com acesso a opções de transporte eficiente e áreas de lazer de acesso público.

Pré requisitos (LL) : Não há

Créditos (LL):

LL 1. Desenvolvimento em Bairro certificado LEED [OU LL2 a LL6]

LL 2. Seleção do Terreno

LL 3.1. Localização Preferencialmente Desenvolvida,

LL 3.2. Localização Preferencialmente Desenvolvida, média [OU]

LL 3.3. Localização Preferencialmente Desenvolvida, superior [E/OU]

LL 4. Infraestrutura de Água e Saneamento Básico

LL 5.1. Proximidade a Recursos Comunitários e 
Transporte Público, básica

LL 5.2. Proximidade a Recursos Comunitários e Transporte Público, média [OU]

LL 5.3. Proximidade a Recursos Comunitários e Transporte Público, superior [OU]

LL 6. Acesso a Espaço Aberto

\section{Categoria TERRENOS SUSTENTÁVEIS (SS)}

A categoria desencoraja o desenvolvimento em terras anteriormente subdesenvolvidas; minimiza o impacto de um edifício sobre os ecossistemas e cursos d'água; incentiva o paisagismo regional apropriado; controles de escoamento de águas pluviais, medidas para redução da erosão, da poluição luminosa, do efeito ilha de calor.

Pré requisitos (SS) :

SS 1.1. Implantar um plano de controle da erosão, sedimentação e poeira na atividade da construção

SS 2.1. Não utilizar plantas invasoras no paisagismo

Créditos (SS):

SS 1.2. Minimizar a intervenção do terreno

SS 2.3 Limitar a área de plantio do gramado [E/OU]

SS 2.4 Implantar espécies que apresentem baixo consumo de água [E/OU]

SS 2.5 Reduzir a demanda de água potável na irrigação em pelo menos $20 \%$ [OU]

SS 3. Redução de llha de Calor - Área de Pisos e Coberturas

SS 4.1. Terreno permeável

SS 4.2 Controle de erosão permanente

SS4.3 ControleeGerenciamento de Águas pluvias-cobertura

SS 5. Controle de Pragas e Doenças sem Produtos Tóxicos

SS 6.1. Implantação Compacta_Densidade moderada

SS 6.2. Implantação Compacta _Densidade alta [OU]

SS 6.3. Implantação Compacta_Densidade muito alta [OU]

\section{Categoria GESTÃO DA ÁGUA (WE)}

A categoria incentiva o uso mais inteligente de água dentro e fora da edificação. A redução do consumo de água é geralmente obtida através de aparelhos mais eficientes, instalações e equipamentos adequados e paisagismo apropriado ao local.

Pré requisitos (WE): Não há

Créditos (WE):

WE 1.1. Sistema de aproveitamento de águas pluviais

WE 1.2. Sistema de reuso de água cinzas

WE 1.3. Utilização do sistema de reuso da concessionária

WE 2.1. Sistemas de Irrigação Eficiente

WE 2.2. Inspeção do sistema de irrigação comissionado [E/OU]

WE 2.3. Reduzir a demanda do sistema de irrigação em pelo menos $45 \%$ [OU]
WE 3.1. Metais sanitários eficientes

WE 3.2. Metais sanitários muito eficientes

\section{E. Categoria EFICIÊNCIA ENERGÉTICA (EA)}

A categoria encoraja uma grande variedade de estratégias para economia de energia: comissionamento, monitoramento de uso de energia; traçado e construção; escolha por equipamentos mais eficientes, adoção de sistemas e iluminação mais eficientes, a utilização de fontes renováveis e limpas de energia geradas no local ou fora do local; e outras estratégias inovadoras.

\section{Pré requisitos (EA):}

EA 1.1. Estabelecer uma performance energética mínima de acordo com etiquetagem "Energy Star for Homes"

EA 11.1. Testes para gerenciamento do gás refrigerante residencial, não uso de CFC's

\section{Créditos (EA):}

EA 1.2. Desempenho Energético Aprimorado

EA 7.1. Distribuição Eficiente de Água Quente

EA 7.2. Tubulação eficiente com isolamento apropriado

EA 11.2. Gerenciamento do gás refrigerante residencial, não uso de CFC's

\section{F. Categoria MATERIAIS E RECURSOS (MR)}

Durante as etapas de construção e operação os edifícios/residências geram grande volume de resíduos e consomem grande quantidade de materiais e recursos. Esta categoria incentiva à seleção de produtos de materiais sustentáveis. Ela promove a redução de resíduos, bem como a reutilização e a reciclagem, e leva em consideração a redução de resíduos na origem de um produto.

\section{Pré requisitos (MR):}

$M R$ 1.1. Especificar materiais eficientes

MR 2.1. Especificar madeiras certificadas FSC

MR 3.1. Implantar um plano de gestão de Resíduos da Construção

\section{Créditos (MR):}

MR 1.2. Sistema construtivo patenteado

MR 1.3. Sistema construtivo de madeira [E/OU]

$M R$ 1.4. Sistema construtivo eficiente[ $E / O U]$

$M R$ 1.5. Sistema construtivo pre-fabricado [OU]

$M R$ 2.2. Materiais Ambientalmente Preferíveis

MR 3.2. Gerenciamento de resíduos da construção

\section{G. Categoria QUALIDADE DO AMBIENTE INTERNO (EQ)}

A categoria promove estratégias que podem melhorar a qualidade do ar interior, bem como proporcionar o acesso à luz natural e vistas e melhorar a acústica.

Pré-requisitos (EQ): 
EQ 2.1. Implantar um plano de controle de Emissão de Gases de Combustão

EQ 4.1. Atender aos parâmetros mínimos de ventilação natural

EQ 5.1. Atender aos parâmetros mínimos de exaustão localizada

EQ 6.1. Atender aos parâmetros mínimos de cálculos vazão do sistema de ar condicionado por ambiente

EQ 9. Atender aos parâmetros mínimos de proteção ao gás radônio

EQ 10.1. Ausência de sistema de ar condicionado na garagem

Créditos (EQ):

EQ 1 Desempenho mínimo de acordo com a etiquetagem "Energy Star for Homes"

EQ 2.2. Aprimorar o plano de controle de Emissão de Gases de Combustão

EQ 3. Controle de Umidade Local

EQ 4.2. Aprimorar o atendimento aos parâmetros mínimos de ventilação natural

EQ 4.3. Teste para desempenho mínimo da qualidade do ar interno

EQ 5.2. Aprimorar o atendimento aos parâmetros mínimos de exaustão localizada

EQ 5.3. Testes para desempenho dos exaustores

EQ 6.2. Testes para desempenho dos controles de climatização

EQ 6.3. Testes para zonas múltiplas do sistema de ar condicionado

EQ 7.1. Filtragem do Ar Exterior_Básico

EQ 7.2. Filtragem do Ar exterior_Moderado

EQ 7.3. Filtragem do Ar exterior_Avançado [OU]

EQ 8.1. Controle de Partículas Contaminantes durante a construção

EQ 8.2. Controle de Partículas Contaminantes durante a ocupação

EQ 8.3. Gestão de Qualidade do Ar, Antes da ocupação

EQ 9.2. Proteção ao radônio_moderada

EQ 10.2. Proteção de Poluentes Provenientes da Garagem

EQ 10.3. Exaustores na garagem [E/OU]

EQ 10.4. Garagem independente ou inexistente [OU]

\section{H. Categoria PRÁTICAS SOCIAIS (AW)}

A categoria estimula as construtoras e profissionais do setor imobiliário a fornecer aos proprietários, inquilinos e gestores de edifícios educação e as ferramentas de que precisam para entender e aproveitar ao máximo os recursos de uma edificação sustentável.

\section{Pré requisitos (AW):}

AW 1.1. Promover conscientização do proprietário para operação e manutenção básica

\section{Créditos (AW):}

AW 1.2. Promover conscientização aprimorada do proprietário para operação e manutenção

AW 1.3. Promover a conscientização pública geral sobre residência

AW 2. Promover a conscientização do gestor do edifício multifamiliar

\subsection{Casos de Estudos}

Considerando que a aplicação dos processos estruturados do sistema norte-americano LEED FOR HOMES na América Latina exige novas atividades de gestão de projeto em relação ao escopo tradicional na construção residencial, em 2015 duas residências pioneiras receberam a certificação ambiental de habitações LEED FOR HOMES no Brasil e Colômbia.

Figura 4: Unidades Residenciais certificadas LEED FOR HOMES.

\begin{tabular}{|c|c|}
\hline Países & $\begin{array}{l}\text { Unidades Habitacionais certificadas } \\
\text { LEED for Homes }\end{array}$ \\
\hline United States & 109.097 \\
\hline Canada & 968 \\
\hline Saudi Arabia (Oriente Médio) & 192 \\
\hline Montenegro ( Europa ) & 70 \\
\hline China, Hong Kong ( Asia ) & 18 \\
\hline Tanzania (África) & 10 \\
\hline Turkey (Europa) & 10 \\
\hline Cayman Islands ( America Latina ) & 3 \\
\hline Brazil ( America Latina ) & 1 \\
\hline Colombia (América Latica) & 1 \\
\hline TOTAL & 110.370 unidades habitacionais \\
\hline
\end{tabular}

Fonte: USGBC - 2016

h t t p:// w w w. us g b c.org/a r t i cle s/ leed-homes-international-market-update-2016 http://www.usgbc.org/advocacy/homes-market-brief

\subsubsection{Caso 1: Projeto Piloto LEED FOR HOMES na Colômbia}

Localizado na cidade de Rio Negro, 17 km da cidade de Medellin; o projeto residencial piloto LEED FOR HOMES da América Latina é a Residência Casa RioNegro na Colômbia http://www.usgbc.org/projects/casa-fenix-rionegro .

O principal conceito de sustentabilidade deste projeto foi o de autonomia no consumo de energia e água apoiada por sistemas automatizados, e uma preocupação da responsabilidade social em relação de população de baixa renda e as gerações futuras em um país sul-americano como a Colômbia. $A$ equipe também observou a oportunidade para implementar estratégias inovadoras na indústria da construção civil e o estabelecimento de uma verdadeira cultura sustentável.

O empreendimento recebeu da organização 
americana United States Green Building Council - USGBC em Fevereiro de 2015, a primeira certificação ambiental internacional de habitações LEED FOR HOMES nível OURO da América Latina. Um dos principais destaques do projeto residencial de 1.800 metros quadrados de área construída, sendo $550 \mathrm{~m}^{2}$ para a residência principal foi a abordagem de integração entre a forma arquitetônica e o entorno através de espaços abertos que permitem a abundância da luz natural, ventilação cruzada e proporcionam vistas às mais belas paisagens do país.

O desempenho no LEED FOR HOMES Piloto Internacional exigiu um processo colaborativo de toda a equipe e um esforço conjunto com os fornecedores, expondo o desafio de se tornar uma referência e um facilitador de mudanças da indústria para a sustentabilidade. Um dos destaques é a utilização do sistema construtivo em estrutura metálica; sistema de energia renovável fotovoltáico; tecnologias de automação inteligente através de um sistema de áudio que anuncia se existem espaços sem pessoas para evitar o desperdício de energia; revestimentos de poliuretano com propriedades acústicas; o uso de vidro temperado e vidros duplos proporcionando conforto ambiental; proximidade a centros comerciais e de saúde, parques, lazer e campos de golfe, proporcionando acesso a muitos serviços básicos e complexos.

\subsubsection{Caso 2: Projeto Piloto LEED FOR HOMES no Brasil}

Localizado em um condomínio residencial Alphaville em Campinas, 115 km da capital financeira de São Paulo; o projeto residencial piloto LEED FOR HOMES no Brasil Residência Alphaville Dom Pedro recebeu da organização americana United States Green Building Council (USGBC) em outubro de 2015, a primeira certificação ambiental internacional de habitações LEED FOR HOMES nível PRATA do Brasil http://www.usgbc.org/projects/ residencia-alphaville-dom-pedro.

Após 16 meses de execução, um dos principais destaques do projeto residencial de $450 \mathrm{~m}^{2}$ está na redução de $60 \%$ no consumo de água, $70 \%$ no consumo de energia, $80 \%$ na geração de resíduos e $30 \%$ nos custos operacionais.

Para a consultoria em conforto ambiental e sustentabilidade LEED FOR HOMES desenvolvida por empresa americana e auditora local brasileira, novas atividades de gestão serviram como oportunidade para práticas ambientais que agregaram valor de qualidade para o empreendimento e garantiram o atendimento aos requisitos da certificação, tais como: sistema construtivo inovador EGOGRID - patenteado pela empresa LCP Engenharia e Construções no Brasil- sendo constituído por painéis de argamassa armada com miolo de EPS (Poliestireno Expandido) intercalados por treliças de aço galvanizado, envoltas nos dois lados por malha do mesmo material, que conferem resistência de 20 a 40 toneladas por metro linear sem a utilização de vigas e pilares, proporcionando excelente isolamento térmico e propriedades termo-acústicas e antifúngicas ; armazenamento de água da chuva em cisterna de 10 mil litros e filtragem por meio de filtros com reutilização de água cinza em descarga e na irrigação eficiente do jardim de paisagismo nativo; metais sanitários eficientes; sistema de distribuição hidráulica com tecnologia de tubos e conexões feitos com PP Polipropilenoe PP-R Polipropileno Copolímero Random; avaliação do desempenho energético determinados em simulação computacional de acordo com a norma internacional ENERGY STAR para a validação da especificação de sistema eficientes; refrigeradores e lavadoras e secadoras de roupas com tecnologia Inverter que permite um consumo até $40 \%$ menor de energia elétrica; especificação de esquadrias de alta qualidade; sistema de placas fotovoltaicas e de aquecimento de água; especificação de madeira legalizada; materiais regionais com alto conteúdo reciclado e baixo índices de Compostos Orgânicos Voláteis - COVs; uso de exaustores para cozinha e banheiros, medidores de $\mathrm{CO}_{2}$ e fumaça, tecnologias que analisam o gás radônio e capacitação do proprietário para manutenção e operação de sua residência sustentável.

\section{CONSIDERAÇÕES FINAIS}

As edificações proponentes a certificação ambiental de habitações LEED FOR HOMES apresentam diferenças consideráveis de gestão de projeto em relação ao escopo tradicional na construção residencial brasileira. A metodologia é complexa e bastante rigorosa, por este motivo obtém valor destacado no mercado. As novas atividades agregam valor de qualidade para o empreendimento com a melhor eficiência energética e hídrica dos sistemas instalados, redução dos custos operacionais, qualidade dos ambientes internos e bem estar do usuário, durante o ciclo de vida da habitação. Questionamentos sobre o reconhecimento da certificação LEED FOR HOMES e o cenário possível de liderança na América Latina, permeiam as discussões.

A certificação LEED for Homes é aplicável a casas e edifícios multifamilares até 20 andares, sendo que 25\% dos empreendimentos certificados são de moradia popular, comprovando a simplicidade do processo amplamente aplicável a habitações de interesse social. A tendência que observamos internacionalmente é a de 
sinergia entre as tipologias LEED, com bairros sustentáveis que possuem moradias certificadas.

A busca de uma certificação ambiental de sustentabilidade internacional LEED outorgada por uma terceira parte independente a uma edificação sustentável na América Latina se apresenta como um caminho para a excelência exigida nos referenciais teóricos, com benefícios ligados às etapas de projeto e construção, através de uma gestão otimizada que garante a obtenção dos melhores resultados com a economia de até $80 \%$ do custo da água, $60 \%$ do custo de energia elétrica, $70 \%$ na geração de resíduos sólidos além de propiciar reduções de perdas de materiais e equipamentos, a segurança e o bem estar dos operários e a durabilidade do produto edificado.

\section{REFERÊNCIAS}

ARBACHE, Ana Paula Org.). Projetos Sustentáveis: estudos e práticas brasileiras I. Editorama : São Paulo, 2010.

GOMES, Vanessa. Avaliação da sustentabilidade de edifícios de escritórios brasileiros: diretrizes e base metodológica. Tese (Doutorado) - Escola Politécnica da Universidade de São Paulo, São Paulo 2003

LCP Engenharia e Construções Brasil - Primeira residência certificada LEED for Homes Silver da América Latina está no Brasil. Site Internet - http://www.lcpconstrucoes.com.br/projeto_campinas.html

MAGRANN Associates Estados Unidos - Residência Alphaville Dom Pedro in Campinas city, Brazil. Site Internet - http://www.magrann.com/magrann_news_results.html?vpage $=0$

United States Green Building Council. Site Internet USGBC - http://www.usgbc.org

United States Green Building Council - LEED FOR HOMES MARKET BRIEF. Site Internet USGBC - http:// www.usgbc.org/advocacy/homes-market-brief

United States Green Building Council - LEED certification update: Third quarter 2016. Site Internet USGBC - http://www.usgbc.org/articles/ leed-certification-update-third-quarter-2016

United States Green Building Council - LEED FOR HOMES USGBC Guide to certification. Site Internet USGBC - http://www.usgbc.org/cert-guide/homes
United States Green Building Council - Casa FENIX Rionegro, Antioquia, Colômbia. Site Internet - http:// www.usgbc.org/projects/casa-fenix-rionegro

\section{United States Green Building Council - Residência Alphaville Dom Pedro, Campinas, Brazil.} Site Internet - http://www.usgbc.org/projects/ residencia-alphaville-dom-pedro

United States Green Building Council - LEED IN MOTION Brasil. Site Internet - http://www.usgbc.org/ resources/leed-motion-brazil

United States Green Building Council - LEED IN MOTION Residential. Site Internet - http://www.usgbc. org/resources/leed-motion-residential

United States Green Building Council - LEED v4 Credits. Site internet - http://www.usgbc.org/v4 